\title{
Halsbandlemming-Reste aus einer Oberen Mittelterrasse des Rheins bei Niederaußem
}

\author{
Von Florian Heller, Erlangen, und Karl Brunnacker, Köln
}

\author{
Mit 3 Abbildungen
}

\begin{abstract}
$\mathrm{Z}$ u s a m me n f s s u ng. Aus der periglazialen Füllung des von einem alten Rheinlauf angelegten Mäanders von Niederaußem (nordwestlich Köln) stammt eine kleine Aufsammlung von Wühlmaus-Resten, und zwar einer Halsbandlemming-Form. Diese unterscheidet sich von den beiden in Europa vorkommenden jungquartären Arten Dicrostonyx henseli HiNron und D.gulielmi SANFORD deutlich durch einfacheren Bau des Kauflächenbildes der Molaren. Auf eine besondere $\mathrm{Be}-$ nennung der offensichtlich neuen Art wurde vorläufig verzichtet. Zwecks Klärung der Frage nach dem zeitlichen Auftreten der Gattung Dicrostonyx im Quartär Europas wurde die einschlägige Literatur kritisch überprüft. Mit Sicherheit darf heute gesagt werden, daß Dicrostonyx bereits zum Bestand vor-würmzeitlicher glazialer Faunen gehört. Die Funde aus dem Quartärprofil von Niederaußem, das Ablagerungen der Riß- oder (wahrscheinlicher) sogar einer noch älteren Eiszeit repräsentiert, sind in diesem Zusammenhang von großer Bedeutung.
\end{abstract}

$\mathrm{Su} \mathrm{m} \mathrm{m}$ a r y . From a depth of 33 meters of one of the Upper Middle Terraces (Obere Mittelterasse) of the Rhine near Cologne comes a small collection of remains of voles all belonging to an arctic-lemming-form, which remarkably differs from the two late Quaternary species of Dicrostonyx henseli HINTON and D.gulielmi SANFORD occuring in Europe by a more simple construction of the enamel pattern of the molars. A name is not yet given to this appartently new species. The respective literature was checked in order to clear the question of the time of appearing of the genus Dicrostonyx in the European Quaternary. It can be said with security that Dicrostonyx in the Quaternary-profile of Niederaußem, representing deposits of the Riss - more probable even of an older glaciation - are in this connexion of great importance.

Auf einer Exkursion des Kölner Geologischen Institutes fand Dipl.-Geol. Dr. E. KEMPF im Bereich des Braunkohlentagebaues Fortuna Nord bei Niederaußem, $20 \mathrm{~km}$ nordwestlich von Köln, Kleinsäugerreste in pleistozänen Ablagerungen. Dr. KEMPF danken wir für die bereitwillige Überlassung seiner Funde. Weiteres Material wurde ausgeschlämmt. Es ist gerechtfertigt, den Fund eingehender zu behandeln: Bislang hat dieser Raum an pleistozänen Säugetierresten nicht viel erbracht. Zudem ist das relativ frühe Auftreten des Halsbandlemmings, das sich aus unseren Untersuchungen ergab, von allgemeinerem Interesse. Dazu kommt die paläoklimatische Bedeutung für die Fundschicht.

\section{Zur Geologie der Oberen Mittelterrassen bei Niederaußem (K. BRUNNACKer)}

Sowohl die Auffassungen über Gliederung und Stratigraphie der Oberen Mittelterrassen (OMT) im allgemeinen wie im besonderen über die Stellung des darin befindlichen Fundpunktes einiger Kleinsäuger bei Niederaußem, auf den sich die folgenden Ausführungen konzentrieren, sind nicht einheitlich. Von Kh. KaIsER (1961) werden drei OMT am Mittel- und Niederrhein ausgeschieden, von L. Ahorner (1962) zwei. Andere Autoren sprechen z. T. nur von einer OMT. Zeitlich wird die OMT-Gruppe der Mindeleiszeit zugeordnet (Kh. KaIser 1961) - wohl deswegen, weil die Untere Mittelterrasse der Saale- (=Riß-) Eiszeit zugerechnet zu werden pflegt. Allerdings könnte nach $\mathrm{H}$. W. Quitzow (nach G. v. D. Brelie 1959) die Unterstufe der Jüngeren Hauptterrasse, die mit Kh. KaISER (1961) der obersten OMT gleichzusetzen wäre, wegen der vom üblichen Typ abweichenden, sandigeren Fazies das Cromerium enthalten und sich nach Süden in den Mosbacher Sanden fortsetzen. Andererseits sollen in einer der OMT (Zitat bei G. v. D. BRELIE 1959) bei Mehlem Frostspalten gefunden worden sein. Für deren Bewertung wäre es allerdings wesentlich zu wissen, ob sie syngenetischer Natur sind.

Bei Niederaußem hat ein alter Rheinlauf einen weit nach Westen ausschwingenden Mäanderbogen geschaffen, in dem anschließend feinkörnige Sedimente abgesetzt wurden. Darauf wurde durch H. W. Qurtzow (1956) aufmerksam gemacht, mit Einstufung als Untere Mittelterrasse. Nach Kh. Kaiser (1961) und L. Ahorner (1962) treten in diesem Bereich dagegen mehrere Stufen der OMT auf. Dieser Auffassung ist zuzustimmen; denn 

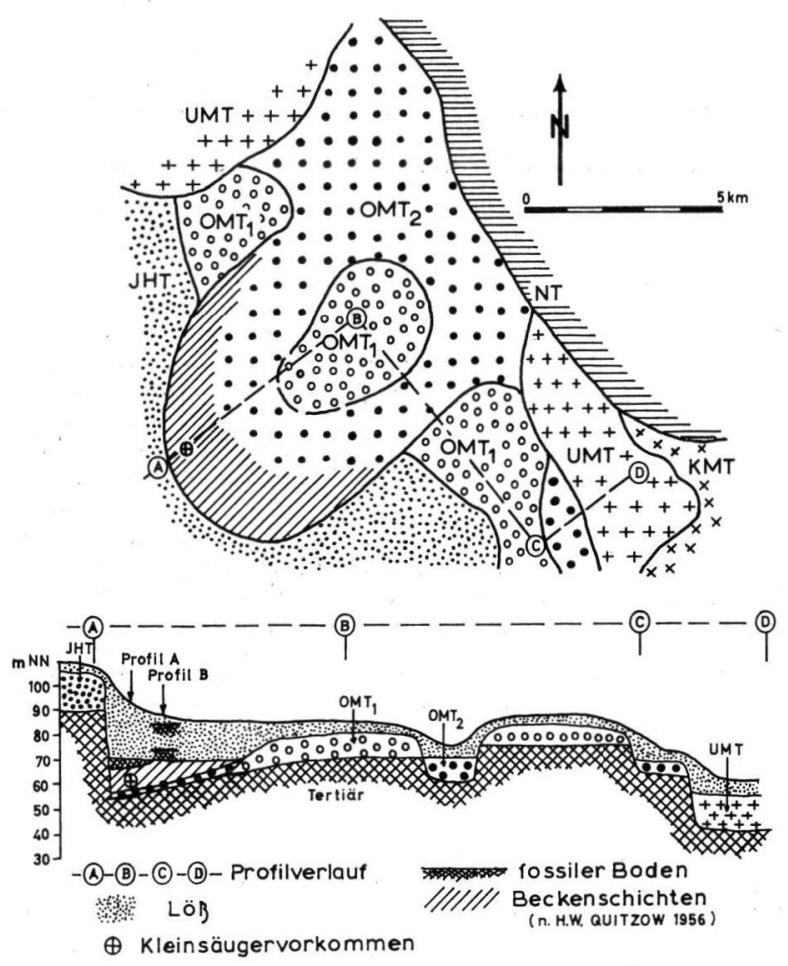

Abb. 1. Die Rheinterrassen bei Niederaußem (umgezeichnet nach H. Breddin 1958) mit Lage der Fundstelle von Dicrostonyx sp.

$\mathrm{JHT}=$ Jüngere Hauptterrasse, $\mathrm{OMT}_{1}=$ ältere Obere Mittelterrasse, $\mathrm{OMT}_{2}=$ Jüngere Obere Mittelterrasse, UMT $=$ Untere Mittelterrasse, KMT $=$ Krefelder Mittelterrasse, NT $=$ Niederterrasse.

bei Berücksichtigung der Mächtigkeit der Deckschichten liegt der Sedimentkörper in einem durchschnittlich 10-20 m höheren Niveau als bei der benachbarten Unteren Mittelterrasse (Abb. 1), worauf schon H. BredDin (1958, Abb. 14) hingewiesen hat. Außerdem können die Terrassen von Köln her weitgehend durchverfolgt werden. Wenn der Auffassung von H. Breddin (1958) als der brauchbarsten, weil Angaben über die Höhenlage von Unter- und Oberkante der Schotter enthaltend, in den Grundzügen der Vorzug gegeben wird, dann wurde zuerst eine ältere OMT $\left(\mathrm{OMT}_{1}\right)$ abgelagert. Darauf folgte Tiefenerosion mit Bildung des Mäanders. Anschließend kam es im eigentlichen Strombett außerhalb des Mäanders zur Ablagerung einer jüngeren $\left.\mathrm{OMT}^{\mathrm{T}}\left(\mathrm{OMT}_{2}\right)^{1}\right)$. Der Mäander wurde zugleich mit feinkörnigen Sedimenten verfüllt. Erneute kräftige Tiefenerosion leitete die weitere Entwicklung ein, die zur Ablagerung des Rinnenschotters, der interglazialen Krefelder Schichten, der Unteren Mittelterrasse usw. führte.

\section{a. Lage der Fundstelle im Profil}

Profil A mit der Fundschicht wurde im November 1964 rd. 250 m südlich Garsdorf aufgenommen (Oberkante in $92 \mathrm{~m} \mathrm{NN}$ ) (Abb. 2):

1) Wegen der Unsicherheiten über die Zahl der Oberen Mittelterrassen sind die hier verwendeten Bezeichnungen $\mathrm{OMT}_{1}$ und $\mathrm{OMT}_{2}$ als reine Lokalbenennungen für die Umgebung von Niederaußem zu werten. 
Boden-Horizonte Tiefe in $\mathrm{m}$

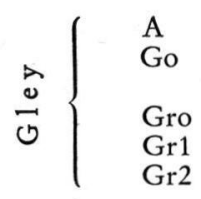

$0-17.00$

$-17.05$

$-17.50$

$-17.90$

$-19.00$

$-19.40$

$-20.10$

Go (g11) $\quad-21.00$

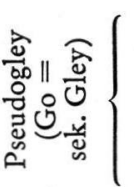

g12 -21.30

g21 -22.20

g22 -24.00

$-24.80$

$-25.50$

$-26.00$

$-27.00$

Löß, Schwemmlöß und Lößlehm, ungegliedert

humushaltiger, dunkelgrauer, feinsandiger Lehm

gelbgrauer, stark feinsandiger Lehm, stark rostfleckig, an der Obergrenze Lößkindl $(5 \mathrm{~cm} \phi)$

braungrauer, stark feinsandiger Lehm, rostfleckig

blaugrauer, feinsandiger Lehm

blaugrauer, feinsandiger Lehm mit Sandbändern

sehr schwach humushaltiger, violettstichiger, grauer, feinsandiger Lehm

grünlichgrauer, feinsandiger Lehm, gebändert
grünlichgrauer, sandiger, feinsandiger Lehm, stark rostfleckig, frostplattig

hellgrünlichgrauer, sandiger, feinsandiger Lehm

hellgrünlichgrauer, sandiger, feinsandiger Lehm, mit großen

Rostflecken $(10 \mathrm{~cm} \phi)$

feinsandiger Lehm, mit Kiesschnüren, nach unten zunehmend sandiger, großfleckig rostbraun und hellgrau marmoriert

rostbrauner Sand und graubrauner feinsandiger Lehm in Bändern

braungrau und blaugrau gebänderter, feinsandiger Lehm

rostbrauner, stark feinsandiger Lehm und Sand, gebändert

blaugrauer, stark feinsandiger Lehm und sehr schwach humushaltiger, grauer, feinsandiger Lehm, gebändert mit kleinen Fältelungen

$-27.80$

grauer, feinsandiger Lehm, gebändert, schwach gestaucht

$-28.30$

grau und rostbraun gebänderter, stark feinsandiger Lehm

$-33.00$

braun und grau gebänderter, z. T. schwach humushaltiger, feinsandiger Lehm mit Sand; unten mehr braun, oben mehr grau

-35.00 humushaltiger, grauer, feinsandiger Lehm, gebändert; nahe der Obergrenze Holzsplitter und Kle ins äugerzähnchen (nestartig angereichert)

-38.00 blaugrauer, toniger Lehm, mit braungrauen Sandbändern $(2 \mathrm{~cm}$ dick)

-38.20 humushaltiger, dunkelgrauer, sandiger Lehm

-38.50
-38.70

sehr schwach humushaltiger, grauer, sandiger Lehm

$\left\{\begin{array}{l}\mathrm{A} \\ \mathrm{AGr} \\ \mathrm{Gr} \\ \mathrm{d} \\ \mathrm{c} \\ \mathrm{c} \\ \mathrm{Gr}\end{array}\right.$

tionen

$-42.00$

grauer, lehmiger Sand bis Sand, nach unten zunehmend kiesiger, nächst Basis einzelne Blöcke (bis $1 \mathrm{~m} \phi$ )

Liegendes: Braunkohlenton (Tertiär).

Daraus ergibt sich:

1. An der Aufnahmestelle wird die Folge durch Schotter eingeleitet, die nach oben in Sand übergehen. Nächst der Basis sind bis $1 \mathrm{~m}$ große Blöcke aus Gesteinen des Schiefergebirges und aus Braunkohlenquarzit angereichert. Sie blieben bei der vorangegangenen fluviatilen Ausräumung älterer Schotterlager als Erosionsrelikte liegen. Wie Abb. 1 zeigt, handelt es sich um eine Schotterlage, die am Gleithang des Mäanders abgesetzt wurde, als dieser sich zunehmend weiter nach Westen verschob. Die Schotter sind deshalb in sich auch nicht völlig gleichalterig. Abgeschlossen wird die geringmächtige kiesig-sandige Lage durch einen Grundwasserboden (Obergrenze $38.00 \mathrm{~m}$ Tiefe). Darin zeichnet sich eine Sedimentationspause, wohl geringerer Wertigkeit, $a b$.

2. Darüber kommen vorwiegend graue, z. T. humushaltige, feinsandige, z. T. tonige Lehme, unten mit Sand- und Kiesschmitzen (35-38.00 m Tiefe). Aufgearbeitetes und umgelagertes Braunkohlentertiär kann hinsichtlich der organischen Komponente nicht völlig ausgeschlossen werden. Dieser Absatz läßt sich am ehesten als Altwasserbildung deuten, die durch eine Rückverlegung des Rheins in das Hauptstrombett ermöglicht wurde.

3. Der nächst höhere Schichtenkomplex (35-20.20 m Tiefe) besteht aus grauen bis gelblichen, dünn gebänderten, feinsandig-schluffigen, z. T. humushaltigen Lehmen mit 
Profil A

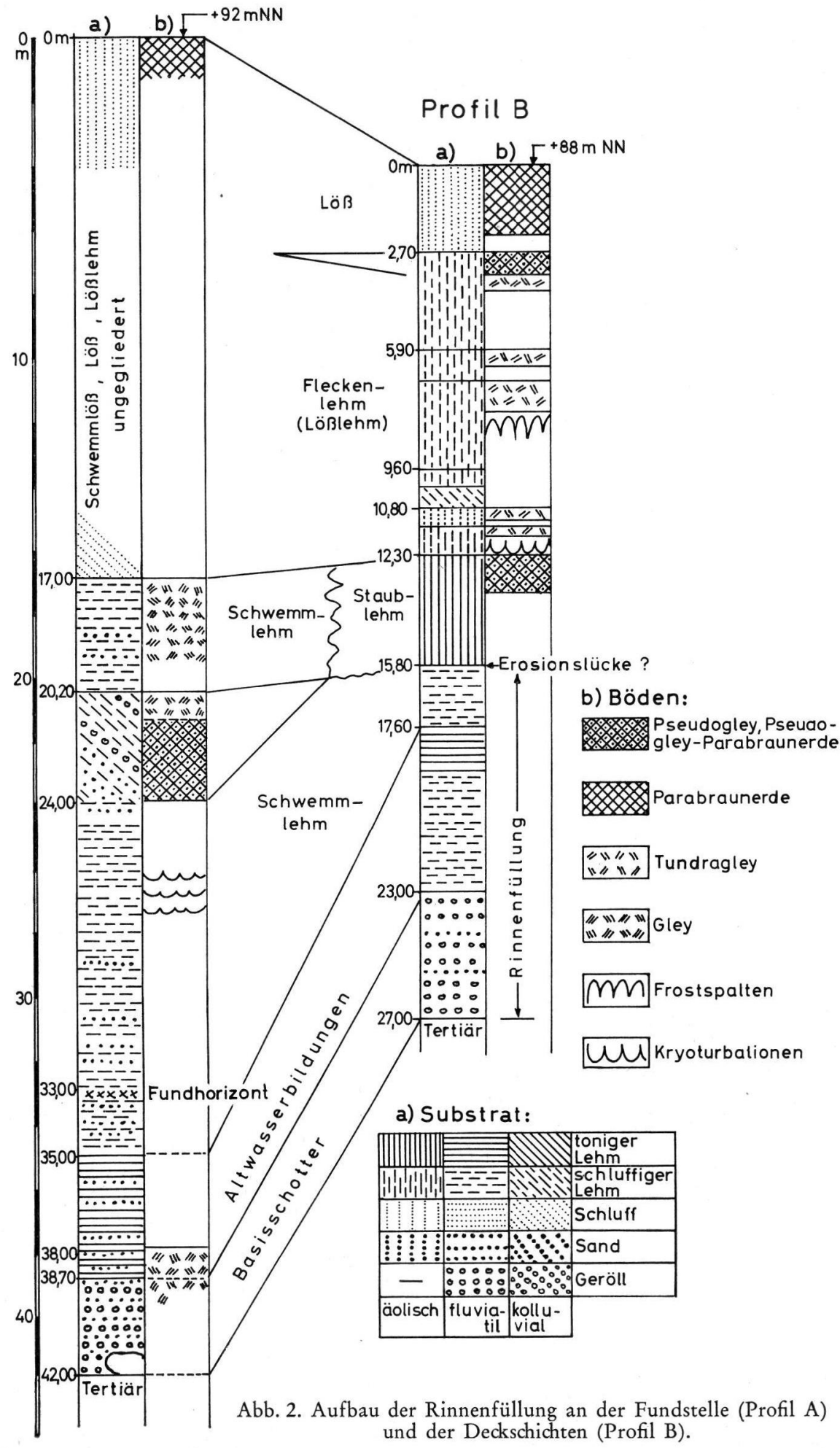

und der Deckschichten (Profil B). 
zwischengeschalteten Sand- und Kiesschmitzen. Darin befindet sich die Fundstelle der Kleinsäuger (in $33.00 \mathrm{~m}$ Tiefe).

Inwieweit kleine Fältelungen in den Lehmen in $26-27.80 \mathrm{~m}$ Profiltiefe $\mathrm{kr}$ y o t u r $\mathrm{b}$ a t e r $\mathrm{N} \mathrm{a} \mathrm{t} \mathrm{u} \mathrm{r} \mathrm{sind,} \mathrm{ist} \mathrm{ohne} \mathrm{weitere} \mathrm{Kriterien} \mathrm{nicht} \mathrm{zu}$ entscheiden. Das vorliegende Profilbild ist ebenso gut durch subaquatische Rutschungen erklärbar. Andererseits dürfen die kleinen Dislokationen, auch wenn sie durch Frost erzeugt wurden, nicht überbewertet werden; denn Kryoturbationen i.w.S. haben keinen Dauerfrostboden zur Voraussetzung. Sie sind allein Indikatoren für ein relativ kaltes Klima mit großer Eindringtiefe des Frostes bei entsprechendem Wasserhaushalt im Boden. In problematischen Fällen sollte damit nicht von vornherein operiert werden, zumal es eine reiche Skala von Pseudokryoturbationen in allen Klimazonen gibt (vgl. z. B. Kh. KaIser 1960, K. BrunNaCKer 1963). Im Zusammenhang mit der Auswertung der Fauna dürfte jedoch die Deutung als Periglazialerscheinung zutreffen.

Einzelne diapirartige Aufpressungen des Braunkohlentertiärs reichen bis in die LößDeckschichten hinein (H. Karrenberg \& H. W. Quitzow 1956). Sie sind wenigstens hinsichtlich ihrer endgültigen Formung jüngeren Alters. Entsprechende Erscheinungen kommen übrigens auch im Oberpfälzer Braunkohlenrevier vor (H. TILlmanN \& E. KIRсноск 1954).

Für die feinkörnigen Sedimente im Mäanderbogen findet sich der Hinweis (H. W. Quitzow 1956, H. Breddin 1958), es handele sich um lößartiges Material sowie z. T. um Ablagerungen als Beckenschichten bzw. als Bänderton in einem See. Dem Typ nach tritt im höheren Bereich ein Hochflutlehm auf, zumal für eine echte Seeablagerung der Grad der Vergleyung zu gering ist (oberhalb $35 \mathrm{~m}$ Tiefe). Besser als die Interpretation als Lößmaterial ist die Deutung als fluviatiler, feinsandiger, lehmiger Schluff - ein Produkt chemischer Verwitterung und mechanisch-fluviatiler Aufbereitung. Eine Lößkomponente ist damit freilich nicht ausgeschlossen.

Bei den in der Fundlage der Kleinsäuger vorkommenden $\mathrm{Holzsplit}$ e r n könnte es sich um aufgearbeitetes Tertiärmaterial handeln. Einen humosen Horizont mit Pflanzenresten erwähnt auch H. BrEDDIN (1958). Ob dieser mit dem genannten übereinstimmt, bleibt fraglich. Doch spricht die freundlicherweise von Fräulein Dr. J. PEters, Bonn, vorgenommene Untersuchung der Holzreste für pleistozänes Alter:

„Die kleinen Astholz-Bruchstücke von wenigen $\mathrm{cm}$ Länge und höchstens $1 \mathrm{~cm}$ Breite sind - wohl infolge starker Pressung - weitgehend abgeflacht. Der Vergleich unter der Lupe und die mikroskopische Prüfung mehrerer dieser Holzreste hatte stets dasselbe Ergebnis:

Zerstreutporiges Laubholz mit einreihigen, heterogenen Markstrahlen und einfachen Gefäßdurchbrechungen. Gefäße zahlreich, die Kreuzungsfelder ihrer Radialwände mit den Markstrahlen mit mehreren einfachen Tüpfeln: Weide = Salix (die Artbestimmung ist bei Weiden nicht möglich).

Die Weide ist u. a. ein Bestandteil der Weichholzaue im Hochflutbett der Flüsse. Die Holzdiagnose als solche kann hier keinen Beitrag zur Altersbestimmung der Schicht liefern, da Weiden bereits im Tertiär weit verbreitet waren. Doch spricht m. E. der gute Erhaltungszustand der Holzreste gegen einen längeren Transport wie auch gegen eine Umlagerung aus älteren Schichten.“

4. Die sandreicheren, solifluktiven (?) Hangendschichten, mit etwas häufigeren KiesEinlagen, wurden abschließend durch einen kräftig entwickelten, vor allem durch die Marmorierung auffallenden Pseudogley überprägt (Oberfläche in $20.20 \mathrm{~m}$ Tiefe). Eine leichte Vergleyung hat den Oberboden des Pseudogleys noch einmal überformt. Sekundäre Frostblätterigkeit deutet auf anschließend wieder zunehmend kühlere Verhältnisse.

5. Weitere Lehme mit Vergleyung liegen darüber (17-20.20 m Tiefe).

6. Dann kommen die eigentlichen, an dieser Stelle hinsichtlich einer Gliederung nichts erbringenden periglazialen Deckschichten. 
Daraus ergibt sich am Profil A folgende Hauptgliederung:

6. Lößdeckschichten, ungegliedert

5. Vergleyter Schwemmlehm

4. Pseudogley

3. Schwemmlehm mit Fundschicht

2. Altwasserbildungen

1. Basis-Schotter periglaziale Deckschichten interglazialer Boden

Rinnenfüllung $=\mathrm{OMT}_{2}$ im Haupttal

\section{Liegendes: Tertiär}

Im Verband mit der Rinnenfüllung tritt als interglazialer Boden ein Pseudogley auf. Unsicher ist, ob der darüber lagernde Schwemmlehm noch zur Rinnenfüllung oder zu den hangenden Deckschichten gehört. Eine klare Entscheidung ist wegen der durch die Vergleyung bedingten starken UUberprägung nicht möglich.

Die zeitliche Gleichsetzung der feinkörnigen Rinnenfüllung mit der Hauptakkumulation der $\mathrm{OMT}_{2}$ im eigentlichen Rheintal ist nicht direkt $\mathrm{zu}$ belegen; denn weite Bereiche des Mäanders sind inzwischen abgeräumt, so daß der Nachweis einer evtl. Verzahnung der Schotter der $\mathrm{OMT}_{2}$ mit den Lehmen, Schluffen und den lokalen sandig-kiesigen, vor allem von der Jüngeren Hauptterrasse abstammenden Einschaltungen kaum möglich ist.

Schotterpetrographische Kriterien erfordern ebenfalls umfangreicheres Material, als es bislang zur Verfügung steht (R. VINKEN 1959). Immerhin ist die Rundung der Gerölle an der Basis des Profils bemerkenswert gut ausgebildet. Außerdem sind die widerstandsfähigsten Restkomponenten relativ stark angereichert. Dies spricht zusammen mit den oben gebrachten Befunden für eine Einstufung der Schotter im Übergang zu einer Warmzeit. Die darüber liegenden Altwasserabsätze vertreten das restliche Interglazial. Die Schwemmlehme stellen eine Lokalfazies der nachfolgenden kaltklimatischen Akkumulation, der $\mathrm{OMT}_{2}$, dar. Die Stellung der Fundschicht im Verband ist damit entsprechend den derzeitigen Möglichkeiten als zur $\mathrm{OMT}_{2}$ gehörig festgelegt.

b. Stratigraphische Einstufung der Fundschicht

Die stratigraphische Position der Fundschicht kann an sich sowohl über die Deckschichten wie über die Terrassen abgeleitet werden.

1. Deckschichtengliederung

Zur Gliederung der Deckschicht wurde an einer geeigneteren Stelle ein weiteres Profil untersucht. Dieses Profil B wurde im Juni 1965 rd. 600 m nordöstlich von A aufgenommen (Oberkante in $88 \mathrm{~m} \mathrm{NN}$ ) (Abb. 2):

Tiefe in $\mathrm{m}$ :

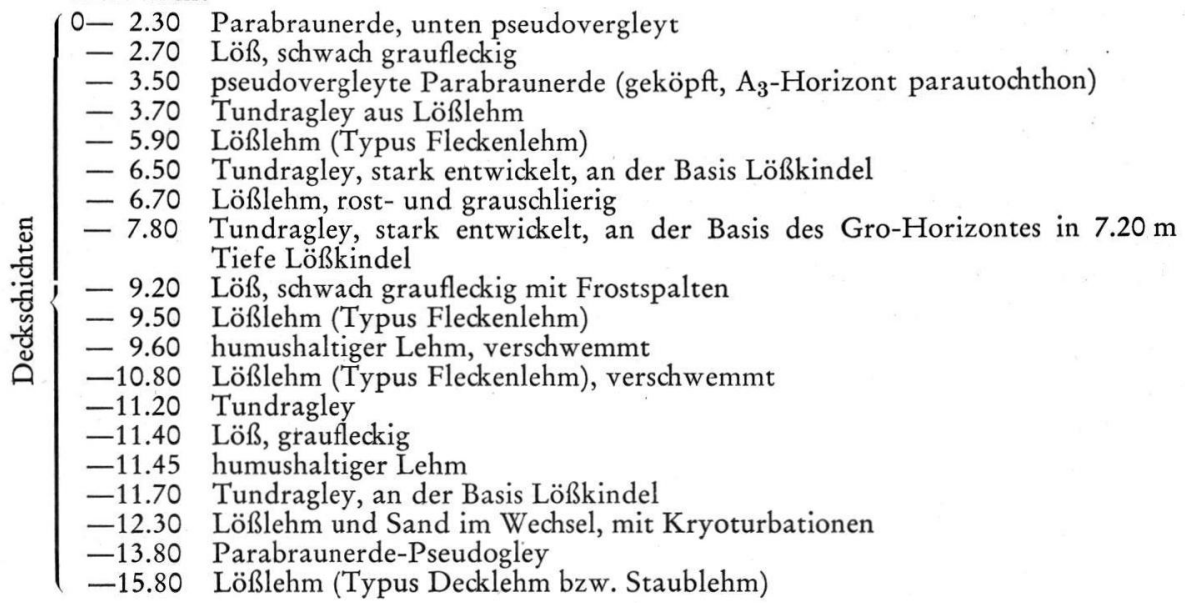


Erosionsdiskordanz?

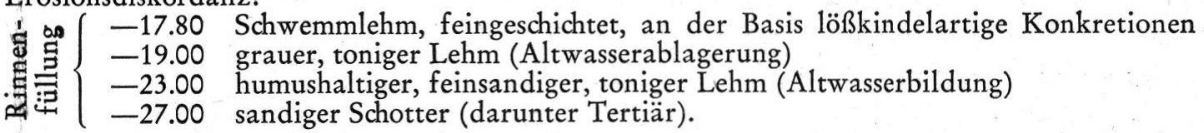

Im Profil B ist eine Deckschichtenfolge mit 3 Lößgliedern erfaßt, die faziell mit der Ausbildung anderer Profile dieses Raumes übereinstimmt (Staublehm - Fleckenlehm Löß), unterteilt durch zwei Interglazialböden. Für die vorletzte Kaltzeit ist, wie in Süddeutschland, das Vorkommen z. T. sehr ausgeprägter Tundragleye typisch.

Gemäß Profil A kann nur gesagt werden, daß der fossilführende Horizont älter sein muß als das Interglazial, das durch den Pseudogley angezeigt wird. Im Profil B, dessen tieferer Aufbau dem des Profils A entspricht, liegen in den Deckschichten zwei eindeutige interglaziale Böden. Entsprechend älter muß die Fundschicht sein. Die Verknüpfung der fossilen Böden der Profile A und B ist auf verschiedene Weise denkbar. Zu berücksichtigen ist dabei, daß eine Flachlandschaft vorlag, in welcher bei geringsten Niveauunterschieden terrestrische Böden (z. B. Parabraunerden und Pseudogleye) von semiterrestrischen ( $\mathrm{z}$. B. Gleye) abgelöst zu werden vermochten. Ein Modellbeispiel dafür ist in der Ziegeleigrube in Wegberg aufgeschlossen. Die scheinbar komplizierte Verknüpfung der Paläoböden und Deckschichten gemäß Abb. 2 fügt sich damit zwanglos in das allgemeine Bild ein.

Das von E. Mückenhausen (1954) beschriebene Profil aus einem heute abgebauten Bereich der Braunkohlengrube Fortuna Nord in ähnlicher geologischer Position beginnt an der Basis mit einem sehr kräftig entwickelten Pseudogley. Innerhalb der darüber folgenden Deckschichten liegen drei weitere Pseudogleye, die als interglaziale Böden angesprochen werden können. Jedoch läßt sich dieses Profil mit den unserigen nicht mehr eindeutig verbinden. Allein die Beschreibung von E. MüCKenhausen (1954) zugrundegelegt, könnte dessen Profil von der OMT 1 stammen (zumal die Schotteroberkante bei rd. $80 \mathrm{~m}$ NN zu veranschlagen ist).

\section{Terrassengliederung}

Beim derzeitigen Kenntnisstand über die Terrassengliederung ist ebenfalls keine endgültige Beurteilung möglich - dafür gibt es noch zu viele Unklarheiten innerhalb der Mittelterrassen. Folgende Einstufung, begrenzt auf den Kölner Raum, ist diskutabel (eine nähere Begründung wird bei anderer Gelegenheit gebracht):

Hauptakkumulation von Niederterrasse $(\mathrm{NT})=$ letzte Kaltzeit

$$
\begin{aligned}
& \text { Krefelder Mittelterrasse }(\mathrm{KMT})=\text { vorletzte Kaltzeit } \\
& \text { Untere Mittelterrasse }(\mathrm{UMT})=\text { drittletzte Kaltzeit } \\
& \text { Jüngere Obere Mittelterrasse }\left(\mathrm{OMT}_{2}\right)=\text { viertletzte Kaltzeit. }
\end{aligned}
$$

An der Basis der UMT liegen die interglazialen Krefelder Schichten. An der Basis der $\mathrm{OMT}_{2}$ liegen im Mäanderbogen ebenfalls Sedimente, die als Interglazial gedeutet werden. Wie andernorts zeigt sich also auch hier an der Wende der Kalt- zu den Warmzeiten eine kräftige Taleintiefung an. Zusammenfassend ergibt sich für die Fundschicht von Niederaußem bei einfachster Interpretation der Deckschichten in Profil A eine Einstufung mindestens in die vorletzte Kaltzeit. Bei Berücksichtigung des Profils B ist eine Einordnung mindestens in die drittletzte Kaltzeit möglich. Einiges spricht jedoch für die Einstufung in die viertletzte Kaltzeit, für die z. B. auch in der Ziegeleigrube Simons, Wegberg, eindeutige Kryoturbationen und Frostspalten kennzeichnend sind.

\section{Die Dicrostonyx-Funde (Fl. Heller)}

Ein bei Niederaußem erschlossenes Quartärprofil lieferte im November 1964 aus einer Tiefe von 33 Metern Reste von Kleinsäugern, die mir Kollege BrunNacker zur näheren Bestimmung und allenfallsigen Außerung hinsichtlich ihres mutmaßlichen geologischen 
Alters übermittelte. Bei der an und für sich relativ kleinen Ausbeute handelte es sich leider nur um recht dürftiges Material von fossilen Wühlmäusen (Arvicoliden), das sich wie folgt zusammensetzte:

2 winzige Fragmente von Mandibeln,

Schmelzbeläge und 1 Fragment von 7 Unterkieferschneidezähnen,

Fragmente von etwa 8 Oberkieferschneidezähnen,

7 Molarenfragmente,

1 intakter $\mathrm{M}^{3}$,

$1 \mathrm{M}_{1}$-Fragment (vorderer Teil),

ferner der Schaft eines Humerus.

Das $\mathrm{M}_{1}$-Fragment, sowie der einzige vollständige Oberkiefermolar gehörten zweifelsohne einer Dicrostonyx-Art an. Die Länge und Beschaffenheit der besterhaltenen Schmelzbeläge von Unterkieferincisiven wiesen ebenfalls auf einen mittelgroßen Vertreter der Arvicoliden hin, als der etwa Dicrostonyx (Halsbandlemming) oder Lemmus (Berglemming) in Frage kommen konnte. Gleiches mochte ferner mindestens für die Mehrzahl der Oberkieferschneidezahn-Reste gelten.

Wenn damit auch der glaziale Charakter der Faunula einigermaßen gesichert erschien, so mußte doch eine genauere Altersdatierung der Fundschicht zunächst noch unterbleiben.

Auf meine Bitte hin, nach Möglichkeit weiteres Fundmaterial zu beschaffen, erhielt ich im Januar 1965 eine zweite Sendung von Wirbeltierkleinfauna, wiederum allerdings nur Reste von Wühlmäusen, die aus ca. $60 \mathrm{~kg}$ Gesteinsmaterial ausgeschlämmt worden waren. Der Erhaltungszustand dieser Ausbeute war erfreulicherweise wesentlich günstiger. Nach entsprechender Präparation und Tränkung mit Zaponlack stehen nunmehr neben verschiedenen wertlosen Fragmenten nicht nur einige vollständigere isolierte Schneidezähne und Molaren, sondern auch 3 Unterkieferfragmente mit einem Teil der Bezahnung zur Verfügung. Sie bestätigten durchaus die erste Bestimmung und zeigen, daß die Wühlmausfaunula von Niederaußem, jedenfalls nach den sicher erkennbaren Resten, praktisch nur Vertreter der Gattung Dicrostonyx umfaßt.

\section{a. Dicroston $y x$ sp.}

Untersuchungsmaterial: 2 r. Unterkieferfragmente mit $\mathrm{M}_{1}$ und $\mathrm{M}_{2} ; 11$. Unterkieferfragment mit $M_{1}$ und dem Rest des $M_{2} ; 1$ r. $M_{1} ; 1$ r., $21 . M_{1}$-Fragmente; 1 weiteres $\mathrm{M}_{1}$-Fragment; 1 r., 1 1. $\mathrm{M}_{3}$; 1 r., 2 1. $\mathrm{M}^{1}$; 1 r., 2 1. $\mathrm{M}^{2} ; 2$ r., 2 1. $\mathrm{M}^{3}$.

An der Zugehörigkeit all' dieser aufgezählten Reste zur Gattung Dicrostonyx kann auf Grund des charakteristischen Kauflächenbildes, d. h. Zahl der Schmelzprismen (7) zwischen vorderem Kopf und hinterer Querschleife am $M_{1}$, Fehlen jeglicher Zementeinlagerungen in den Einbuchtungen der Schmelzdreiecke und Auftreten winzigkleiner zusätzlicher Schmelzschlingen an bestimmten Zähnen ( $\mathrm{M}^{1}$ und $\mathrm{M}^{2}$ : Hinterende außen; $\mathrm{M}_{2}$ : Vorderende innen) nicht der geringste $Z_{w}$ eifel bestehen. In der Ausbildung des $\mathrm{M}_{1}$ (Abb. 3 a-c), d. h. der Gestaltung des vorderen Kopfes, herrscht die übliche Variabilität, wie sie vor allem von den jungpleistozänen Vertretern der Gattung (Dicrostonyx henseli Hinton und D. gulielmi S ANFORD) bekannt ist, weshalb jedenfalls nach diesem Gesichtspunkt allein eine Unterscheidung weiterer Arten nicht nur unmöglich, sondern sogar völlig sinnlos wäre.

Bezüglich des Aussehens von $\mathrm{M}^{1}$ und $\mathrm{M}^{2}$ (Abb. $3 \mathrm{~g}, \mathrm{~h}_{1}$ bzw. $3 \mathrm{i}, \mathrm{k}$ ) muß festgestellt werden, daß bei sämtlichen vorliegenden Exemplaren die hintere Wand des letzten in n e ren Schmelzdreieckes kaum jemals wirklich konkaven Verlauf aufweist und sich daher auch keine Spur eines kleinen inneren Zusatz-Dreieckes findet. Die Zähne erinnern deshalb auffallend stark an ihr Pendant bei der fossilen Art Dicrostonyx benseli und 


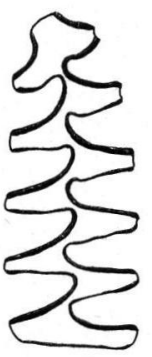

a. $1 . M_{1}$

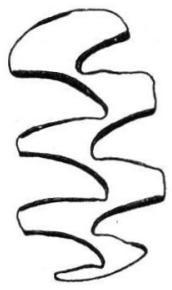

h. $r . M^{1}$

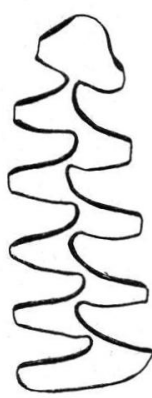

b. r. $M_{1}$

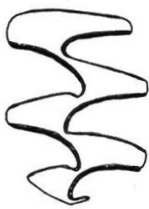

i. $1 . \mathrm{M}^{2}$

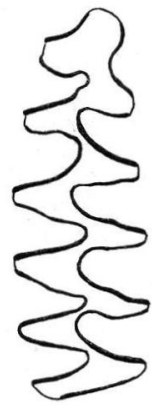

c. r. $M_{1}$

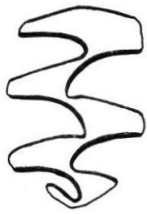

k. r.M $M^{2}$

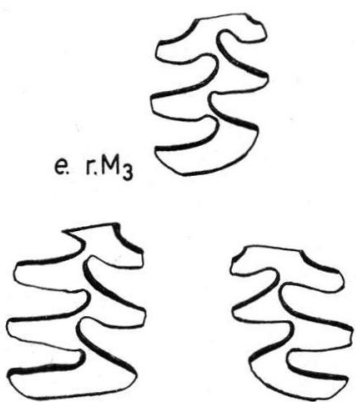

f. $1 . M_{3}$

g. I.M

d. $\mathrm{r} \cdot \mathrm{M}_{2}$
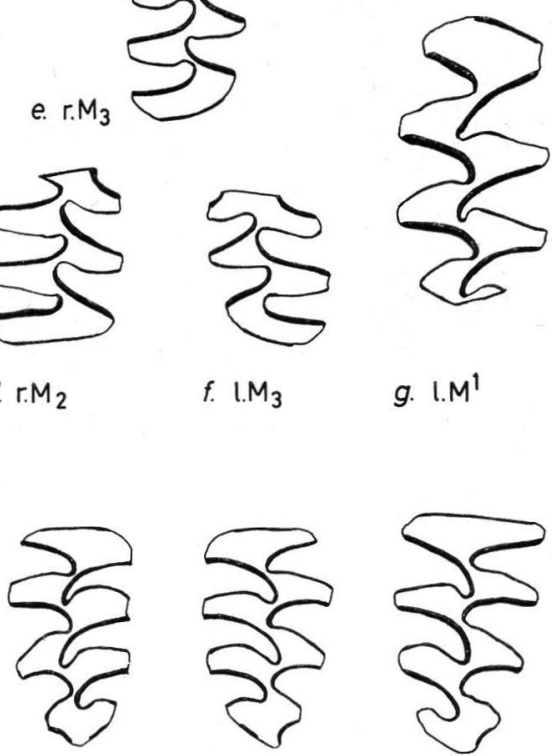

l. r. $\mathrm{M}^{3}$

m. $1 . M^{3}$

ก. $1 . M^{3}$

Abb. 3. Molaren-Kauflächenformen von Dicrostonyx sp. von Niederaußem. x 10.

dem rezenten Vertreter D. hudsonius Pallas. Dies um so mehr, als auch das Verhalten des Schmelzbandes am hintersten inneren Schmelzdreieck hinsichtlich seines Dickenverhältnisses Ähnlichkeit mit den beiden genannten Arten aufweist.

Auffallend an dem r. M2 (Abb. $3 \mathrm{k}$ ) ist die starke Schrägstellung bzw. Rückbiegung seiner kleinen äußeren Zusatz-Schlinge, die sonst bei den rezenten und fossilen Arten im allgemeinen mehr oder weniger horizontale Stellung zeigt, was auch für eines der mir vorliegenden Exemplare gilt. Der gut erhaltene rechte $\mathrm{M}_{2}$ (Abb. $3 \mathrm{~d}$ ) besitzt ebenso wie ein zweites Exemplar an seiner Außenseite vorn höchstens eine leichte Andeutung eines zusätzlichen Schmelzgebildes. Das innere kleine Schmelzdreieck erweist sich abweichend von der üblichen Ausbildung bei den rezenten und fossilen Arten als regelrecht spitz auslaufend.

Besonders wichtig und für eine allenfallsige Klärung der verwandtschaftlichen Beziehungen geradezu entscheidend, erscheint mir nun das Kauflächenbild des $\mathrm{M}_{3}$. Bei der fossilen Art D. gulielmi findet sich im allgemeinen an diesem Zahn e in P a a r (also je ein äußeres und inneres) jener kleinen zusätzlichen Schmelzdreiecke (vestigial angles Hintons), von denen schon mehrfach die Rede war. Die ebenfalls fossile Art D. henseli besitzt dagegen nur e in inneres kleines Schmelzdreieck. Bei der rezenten Art D. hudsonius schließlich fehlen sogar beide, und das gleiche Aussehen zeigen die zwei uns vorliegenden Exemplare dieser Zahnkategorie (Abb. 3 e,f). Damit nähern sich also die aus der oberen Mittelterrasse von Niederaußem stammenden Dicrostonyx-Reste wiederum sehr stark der heute noch existierenden Art D. hudsonius. Trotzdem möchte ich aber keineswegs an eine völlige Identifizierung mit letzerer denken. Vermutlich liegt eine bisher unbekannte Form vor. Unter Berücksichtigung der etwas spärlichen Reste aus der neuen Fundstelle ziehe ich es vor, noch keine nähere Artbestimmung vorzunehmen, sondern besseres und umfangreicheres Material abzuwarten. 
Hingegen ist es wohl an der Zeit, sich einmal grundsätzlich über die Bedeutung der kleinen, „spurenhaften“ Schmelzdreiecke an den Molaren $\mathrm{M}^{1}, \mathrm{M}^{2}, \mathrm{M}_{2}$ und $\mathrm{M}_{3}$ der verschiedenen Dicrostonyx-Formen klar zu werden. Hinton (1926, S. 147) sah in ihnen alte Reststrukturen oder Rudimente, daher die Bezeichnung vestigial angles, während ALLEN (1919, nach Hinton 1926) mehr an sich anbahnende Komplikationen, also an Orimente dachte. Solange keine geologisch älteren fossilen Formen von Dicrostonyx vorlagen, war eine richtige Entscheidung m. E. nicht möglich. Die neuen Funde weisen hingegen darauf hin, daß die "vestigial angles" doch wohl eher im Sinne Allens zu deuten sind, wir also als Vorläufer der jungpleistozänen Arten D. gulielmi und benseli Formen mit wesentlich einfacher gestalteten Molaren erwarten müssen.

\begin{tabular}{|c|c|c|c|c|c|}
\hline & $\mathrm{Maß}$ & $\mathrm{nm})$ der $D i c$ & on $\mathrm{Nie}$ & & \\
\hline & Länge & Breite & & Länge & Breite \\
\hline r. $M_{1}$ & 3,8 & 1,3 & r. M1 & 2,7 & 1,4 \\
\hline r. $\mathrm{M}_{1}$ & 3,8 & 1,3 & 1. M1 & 2,7 & 1,4 \\
\hline r. $\mathrm{M}_{1}$ & 3,5 & - & 1. $\mathrm{M} 1$ & 2,7 & 1,4 \\
\hline 1. $\mathrm{M}_{1}$ & 3,4 & 1,4 & r. $\mathrm{M}^{2}$ & 2,0 & 1,4 \\
\hline r. $\mathrm{M}_{2}$ & 1,7 & 1,3 & 1. $\mathrm{M}^{2}$ & 2,0 & ca. 1,5 \\
\hline r. $\mathrm{M}_{2}$ & 1,6 & 1,3 & 1. $\mathrm{M}^{2}$ & 1,9 & 1,4 \\
\hline 1. $\mathrm{M}_{3}$ & 1,5 & 1,3 & r. $\mathrm{M}^{3}$ & 2,1 & 1,3 \\
\hline r. $\mathrm{M}_{3}$ & 1,6 & $1,2-1,3$ & r. $\mathrm{M}^{3}$ & 2,3 & 1,3 \\
\hline & & & 1. $\mathrm{M}^{3}$ & 2,1 & 1,3 \\
\hline & & & 1. $\mathrm{M}^{3}$ & 2,3 & 1,4 \\
\hline
\end{tabular}

b. Die isolierten I inf. und sup. von mittelgroßen Arvicoliden Untersuchungsmaterial: 9 r., 5 l. I inf., bzw. Fragmente; 10 r., 7 l. I sup.

Daß die vorliegenden vollständigeren I inf. der Gattung Dicrostonyx angehören, läßt sich unschwer an Hand der bezahnten Unterkiefer nachweisen. Gleichzeitig stimmen die Zähne von Niederaußem auch nach Größe und Krümmung weitgehend mit fossilem Material von Dicrostonyx aus dem Jungpleistozän überein. Immerhin scheinen gewisse Unterschiede und zwar hinsichtlich etwas größerer Höhe und Breite = Dicke gegenüber meinen Vergleichsstücken zu bestehen, was auf spezifischer Verschiedenheit beruhen dürfte.

Aus Billigkeitsgründen und auch wegen der Häufigkeit wird man versucht sein, die unter sich gleichgestalteten Oberkieferschneidezähne ebenfalls mit Dicrostonyx in Verbindung zu bringen. Gegenüber jungquartären Vergleichsstücken erscheinen sie etwas zierlicher und auch etwas stärker gekrümmt. Sie erinnern in mancher Hinsicht an die Zähne der großen Microtus-Arten, weshalb bei der Bestimmung eine leichte Unsicherheit aufkommt, welcher Gattung man sie endgültig zuweisen darf.

c. Das zeitliche Auftreten der Lemminge im Quartär Europas

Unter den in quartären Ablagerungen Europas vorkommenden Wühlmäusen erfreuen sich die Lemminge, d.h. die Vertreter der Gattungen Dicrostonyx GLoger 1841 und Lemmus LiNk 1795, seit jeher besonderer Beachtung. Auf Grund ihrer heutigen circumpolaren Verbreitung - Dicrostonyx, mit Ausnahme der zwischen dem 53. und 54. nördl. Br. gelegenen Aleuten-Insel Unalaschka, nicht südlicher als bis $68^{\circ}$; Lemmus mit einigen Arten bis 56 und sogar $52^{\circ}$ vorstoßend - gelten sie als charakteristische Kaltformen, deren Aussagefähigkeit in klimatischer Hinsicht zumindest ab Jungquartär unbestritten ist. Von allen Arvicoliden morphologisch am meisten aberrant, liegt trotz relativ guter Kenntnis der Entwicklungsgeschichte der Gesamtfamilie gerade ihre Herkunft und Ableitung noch sehr im Dunkel. Noch 1926 wußte Hinton (S. 138 und 158) über das fossile Vorkommen von Dicrostonyx nur zu berichten, daß fossile Reste von mindestens 2 Arten in den spätpleistozänen Ablagerungen von West- und Mitteleuropa häufig vorkommen und auch aus Nord- und Zentralasien bekannt geworden seien. In 
ähnlicher Weise äußerte sich der genannte Autor (S. 195) über die quartären LemmusFunde, welche, jedenfalls in Europa, im allgemeinen mit der Art Lemmus lemmus L. in Verbindung gebracht würden.

Größeren Raum widmete Stehlin (Dubois et Stehlin 1933, S. 126-128) der Ơkologie, sowie der rezenten und quartären Verbreitung des Halsbandlemmings. Nach seinen Worten ist die „tertiäre Geschichte“ dieser Gattung noch völlig schleierhaft, vielleicht habe sie sich aber irgendwo im Bereich ihres heutigen Verbreitungsgebietes entwickelt. Unter Aufzählung wohl fast aller wichtigeren, bis dahin ermittelten Fundpunkte schreibt STEHLIN weiter (S. 127), daß Vertreter der Gattung Dicrostonyx in den mittleren Breiten Europas nicht v o $r$ dem Moustérien auftauchten und erst gegen Ende der paläolithischen Epoche, im Magdalénien, maximale Häufigkeit erlangten. Diese keineswegs erst auf STEHLIN zurückgehende Erkenntnis fand auch in der Folgezeit immer neue Bestätigung und sichtbaren Niederschlag in verschiedenen Tabellen, die von einem ersten Lemmingvorstoß im Altwürm und einem späteren Hauptvorstoß im Verbande einer "sehr kalten $=$ Tundrenfauna“ zur Zeit des jüngeren oder oberen Würm sprechen.

Unter diesen Umständen dürfte es verständlich sein, daß Verfasser (HELLER, 1930) bei der Auffindung eines einzelnen Unterkieferfragmentes von Lemmus lemmus L. in den altquartären Verfüllungen der Sackdillinger Höhle/Oberpfalz zunächst Bedenken trug, diesen Rest so ohne weiteres als Element der Gesamtfauna anzuerkennen, vielmehr nach Möglichkeiten suchte, jenen „Fremdling“ als Zugabe aus späterer Zeit, etwa durch Umlagerungsvorgänge usw. zu erklären. Demgegenüber konnte BRUNNER (1933) bei neuen Nachgrabungen in der genannten Höhle nochmals, und zwar mehrere Belege von Lemmus in absolut ungestörtem Schichtverband des erschlossenen Profils erbeuten, womit die Gleichalterigkeit der etwas auffallenden Art mit den übrigen Säugerresten einwandfrei erwiesen war. Eine weitere Bestätigung dafür, daß der, oder vielleicht besser ein Berglemming (Lemmus) sehr wohl gewissen, d.h. mehr kaltzeitlichen Altquartärfaunen zugehören kann, erbrachte schon wenige Jahre später die Auffindung entsprechender Reste auch in fossilführenden Spaltenfüllungen von Erpfingen/Schwäb. Alb (HELLer 1936). Seitdem liegen Fundmeldungen eines (oder mehrerer Vertreter?) von Lemmus aff. lemmus altquartärer Faunenzugehörigkeit (z. T. mit Mimomys-Arten) vor aus der Ukraine von Tschortkow b. Tarnopol (Pidoplichko 1955, nach Kretzor 1956 und JAnossy 1961), aus der Höhle C 718 bei Koněprus/ČSR (FEJFAR 1956, S. 95; 1959, S. 32), aus einer zweiten artenreichen Fauna von Erpfingen (HELLER 1958), einer kleinen Gerinneverfüllung von Deinsdorf/nördl. Frankenalb (Heller 1963 a), zu welchen Nachweisen auf Grund bisher noch unveröffentlichten Materials schließlich noch die Spaltenfüllungen von Schernfeld b. Eichstätt/Mfr. (siehe hierzu Deнм 1962) kommen.

Hier ist nun der Vollständigkeit halber nachzutragen, daß Kormos (1937, S. 304) seinerzeit Zweifel äußerte, ob es sich bei den Lemmingfunden in der Sackdillinger Höhle auch tatsächlich um Lemmus lemmus L. handle. Bereits ein Jahr vorher hatte Verf. (HELler 1936, S. 22) selbst schon darauf hingewiesen, daß man allenfalls auch an das Genus Myopus denken müsse, das im Quartär Sibiriens durch eine fossile Art (Myopus brandti TSCHERSKY) vertreten ist. Kormos glaubte bei der von BRUNNER abgebildeten Oberkieferbezahnung gute Übereinstimmung mit dem rezenten Myopus schisticolor feststellen zu können und schloß: „Ich halte es demnach für sicher, daß der rätselhafte Lemming aus dem Windloch (Sackdillinger Höhle) nicht der Gattung Lemmus, sondern dem, in der heutigen Fauna Centralasiens und Sibiriens durch mehrere Arten vertretenen Genus Myopus angehört." Auf diesen Passus bezieht sich wiederum SoEngel (1943, S. 27), wenn er unter Hinweis auf die ungeklärte Frage, ob bei den fossilen Funden Lemmus oder Myopus vorliegt, meint, mit dem Vorkommen von Lemmus im Altdiluvium könne vorerst nicht gerechnet werden. Hierzu ist aus neuerer Sicht abschließend zu sagen: Es berührt immerhin merkwürdig, daß auch die späteren Autoren, die sich, wie oben erwähnt, 
bei der Bestimmung ähnlicher Funde vor die gleiche Frage gestellt sahen wie Verfasser, sich jeweils zugunsten von Lemmus lemmus entschieden.

Wie aber steht es nun um das Vorkommen von Dicrostonyx-Resten in einwandfrei älteren als würmzeitlichen Ablagerungen? Hier müssen wir uns zunächst einmal mit der von BrunNer (1936) aus dem Osterloch bei Wurmrausch (Opf.) beschriebenen und als mitteldiluvial datierten Fauna auseinandersetzen. Obwohl eine klare Schichtentrennung nach der Darstellung des genannten Autors (S. 16) n i ch t möglich war, hat dieser im wesentlichen auf Grund einer unterschiedlichen Knochenfärbung eine Aufteilung der Gesamtfauna in 3 Niveaus versucht. Danach führten nur die mittlere und die obere Lage Dicrostonyx henseli und Lemmus lemmus neben Arten, die keineswegs besonders auffällig sind, sondern in jeder normalen jungquartären würmzeitlichen Fauna vorkommen. Einen Hinweis auf höheres Alter - zumindest der unteren Lage - erblickte BrunNER nun in der Auffindung von 4 Unterkieferresten von Sorex kennardi Hinton, die er erstaunlicherweise kurzerhand als altdiluviale Art bezeichnete (S. 19). Davon kann jedoch keinesfalls die Rede sein. Hinton (1911, S. 538) gibt als locus typicus für seine neue Art Sorex kennardi die III. Themse-Terrasse von Ponder's End an. Als Begleitfauna werden von dort angeführt (Hinton 1926, S. 135): Dicrostonyx henseli (sehr häufig), Microtus anglicus und Formen der Microtus arvalis-Gruppe, also eine Fauna durchaus würmzeitlichen Gepräges. Zudem konnte Verf. (Heller 1932, S. 350/51), was Brunner völlig entgangen zu sein scheint, diese Art, oder wenigstens eine ihr sehr nahestehende Form, zum erstenmal außerhalb Englands aus der Raumgrotte/Hersbrucker Jura im Rahmen einer wiederum einwandfrei jungquartären Tiergesellschaft (mit Dicrostonyx gulielmi, ? D. henseli und Lemmus lemmus) nachweisen. Brunners weiterreichende Schlüsse, die Gesamtfauna des Osterloches müsse „in eine frühe Epoche des Mitteldiluviums gestellt werden“, dürfte daher jeglicher Grundlage und Berechtigung entbehren. Viel zutreffender ist oder wäre wohl eine Einstufung der tiefsten Fundlage jener mehrmals erwähnten Höhle in das ausgehende Riß-Würm-Interglazial oder, vielleicht noch besser, in das Frühwürm.

Die Artenliste der nicht genauer zu gliedernden Ablagerungen aus dem Enzendorfer Loch bei Vorra a. Pegnitz (Brunner 1937) weist in einer typisch jungquartären Fauna neben zahlreichen Exemplaren von Dicrostonyx henseli-(298) und 2 Lemmus lemmusMandibeln zunächst 180 Reste von Microtus nivalinus Hinton (siehe Bemerkungen hierzu etwas weiter unten!), ferner solche von M.gregalis, M.arvalis-agrestis und M.ratticeps aus. Sodann fanden sich einige wenige Belege von Pitymys-Arten, welche mit P.arvaloides Hinton und P.gregaloides Hinton identifiziert wurden. Eben vor allem wegen des Auftretens der beiden letztgenannten Formen argumentiert BRUNNER (S. 50): „Es darf somit das Alter dieser Fauna in eine frühe Stufe des mittleren Diluviums gestellt werden. Beide Arten sind nach den bisherigen Erfahrungen typische Leitfossilien des Altdiluviums." Genügt dieser Befund aber bereits zu derart weittragenden Schlußfolgerungen?

Auch für den Großteil der Fauna (Schicht I-III) aus der Hirtenweberhöhle bei Neunkirchen in der Nähe von Sulzbach/Opf. nimmt Brunner (1939) mitteldiluviales Alter in Anspruch. In Schicht I fehlt Dicrostonyx benseli (noch?), während er in Schicht II mit $0,2 \%$, in Schicht III mit $0,3 \%$ und in der auch von BRunNER als würmzeitlich aufgefaßten Schicht V mit 12\% am gesamten Fossilbestand vertreten ist. Eine eingehendere Begründung, worauf sich die Einstufung der tieferen Schichtglieder des Profils in einen vor-würmzeitlichen Abschnitt des Quartärs stützt, ist Brunner schuldig geblieben. Die Artenliste enthält jedenfalls außer den üblichen, für das Jungquartär charakteristischen Formen lediglich Sorex kennardi HINT. (Schicht III), Chionomys = Microtus nivalinus HINT. (Schichten I-III, sowie V) und Pitymys arvaloides HINT. (Schichten I und II). Bezüglich Sorex kennardi kann auf das bereits weiter oben Gesagte verwiesen werden. Unter Chionomys nivalinus, einer Art, die von HINToN ursprünglich für altquartäre Ver- 
treter aufgestellt wurde, wollte BRUNNER grundsätzlich all jene fossilen Schneemausreste verstanden wissen, die sonst in den Faunenzusammenstellungen als Ch.nivalis MART. erscheinen. Bleibt als einziges Element, welches für vor-würmzeitliches Alter sprechen könnte, noch Pitymys arvaloides übrig. $\mathrm{Zu}$ seiner Bestimmung, welche die betreffenden Reste $\mathrm{zu}$ altquartären bzw. allgemein zu geologisch älteren Elementen stempeln würde, sagt Brunner (S. 439) jedoch selbst: "Vielleicht handelt es sich hier noch um P.subterraneus. Eine sichere Entscheidung kann erst durch eine bessere stratigraphische Differenzierung der Schichten- bzw. Faunenfolge des mittleren Diluviums getroffen werden.“ M. E. liegt keine Veranlassung vor, die Fauna der Hirtenweberhöhle auch nur zum Teil als mitteldiluvial zu betrachten. Vielmehr passen die festgestellten verschiedenen Fundschichten samt und sonders recht gut in die allgemeine Abfolge Frühwürm-Altwürm bis wahrscheinlich Hochwürm.

Eine völlige Klärung der Frage, ob die Gattung Dicrostonyx im Quartär Europas überhaupt bereits v or Anbruch der letzten Eiszeit (Würm) vorkam bzw. in Erscheinung trat, erbrachten auch die späteren Veröffentlichungen BRunNers über fossile Säugetierfaunen aus dem Gebiet des Fränkischen Jura nicht. Er beschrieb zwar noch verschiedentlich Fundplätze von Dicrostonyx benseli (Helmloch, Gaisloch b. Velden, Kleine Teufelshöhle b. Pottenstein, Markgrabenhöhle) oder von D.gulielmi (Helmloch), die er auf Grund des gleichzeitigen Nachweises einzelner, sonst nur aus dem jüngeren Altquartär bekannt gewordenen Arten zumindest für mittel-, teilweise sogar für altdiluvial hielt. (BRUNNER 1940, 1949, 1951 und 1952). Die mitgeteilten Artenlisten kranken leider alle daran, daß nach BRunNer selbst in den betreffenden Höhlen entweder durch natürliche Umlagerungsvorgänge der Sedimente, wie etwa durch Verschwemmungen, eine weitgehende Vermischung von Resten aus den verschiedensten Abschnitten des Quartärs erfolgte, oder daß sich innerhalb der Gesamtmasse der Sedimente keine eigentliche, genauere Schichtentrennung durchführen ließ. Auf alle Fälle ist das gesamte Faunenmaterial dadurch mit gewissen Vorbehalten zu betrachten, womit eine, auch nur einigermaßen einwandfreie stratigraphische Entzerrung und Auswertung sehr erschwert wird, ja geradezu ein Ding der Unmöglichkeit darstellen dürfte.

Auf gewisse Widersprüche, die hinsichtlich der zeitlichen Datierung zuweilen in den Angaben BRUNNERs enthalten sind, sei hier nur am Rande eingegangen.

So wird in einem Falle (Markgrabenhöhle, Brunner 1952) die Fauna im wesentlichen wegen des Vorkommens von Talpa episcopalis Konmos ins Altdiluvium gestellt, während in einem anderen Falle das Auftreten derselben Art in einer würm-interstadialen Fauna (Schmiedberg-Abri, Brunner 1959) nicht als störend empfunden wird.

Das ganze hier eigens aufgezeigte Dilemma rührt nicht zuletzt daher, daß wir trotz jahrzehntelanger intensiver Forschungsarbeit bisher kaum Fundplätze rißzeitlicher Ablagerungen mit reicherer Fossilführung, insbesondere von Mikromammaliern haben, und uns somit die eigentlichen Rißfaunen im Grunde genommen noch ziemlich unbekannt sind. Aus Bayern stammt bisher lediglich die Angabe, wonach im unteren Teil des rißeiszeitlichen Hochterrassenschotters bei Ismaning auch einige Zahnlamellen vom Halsbandlemming gefunden wurden (BrunNacker u. a. 1964, S. 63/64). Hier kann nun vielleicht das mächtige, vielgliederige und vor allem an Knochenresten so überaus reiche Quartärprofil in der Höhlenruine von Hunas-Hartmannshof (HELLER 1963b) endlich einen gewissen Wandel schaffen.

In den kantigen Bruchschuttbildungen der sicher glazialen Phasen zugehörigen Glieder des Schichtenprofils von Hunas konnten sowohl Reste von Lemmus lemmus, wie auch einer Dicrostonyx-Art nachgewiesen werden. Letztere sind zwar keineswegs besonders zahlreich, ihre grundsätzliche Anwesenheit unterstreicht jedoch bestens den allgemeinen Kaltsteppencharakter der betreffenden Fauna, die an weiteren Kleinformen u. a. verschie- 
dene, z.T. erloschene Wühlmausarten, Steppenhamster, Zwergpfeifhasen, große und kleine Wiesel usw. enthält.

Ohne Zweifel hat also nach diesen Ausführungen die Gattung Dicrostonyx als ein echtes Element auch rißzeitlicher Kaltfaunen zu gelten. Das stratigraphisch gesicherte Vorkommen in der Fauna der Höhlenruine von Hunas schlägt aber zugleich auch eine Brücke vom mittleren Quartär zu dem noch früheren Nachweis der Gattung in altpleistozänen Bildungen, womit die betreffenden Funde doch nicht mehr gar so isoliert dastehen, wie es nach den Darstellungen von Janossy (1961, S. 4 u. 8) den Anschein hatte. Die Höhle C 718 bei Koněprus/ČSR schließlich, die schon weiter oben unter den altquartären Fundstellen für Lemmus lemmus angeführt wurde, hat gleichzeitig die bisher ältesten Belege eines Vertreters der Gattung Dicrostonyx geliefert (FEJFAR 1959, S. 32), von dessen richtiger Bestimmung sich JANOsSY überzeugen konnte. Die reiche Begleitfauna von 43 Arten aus der basalsten Schicht enthält an sonstigen Wühlmäusen außer Mimomys savini HintoN und intermedius Newton als den interessantesten Elementen Pliomys episcopalis Mehely und Pl.lenki Heller, sodann die recht bezeichnenden Formen Pitymysarvaloides Hinton, P.hintoni Kretzor, P.gregaloides Hinton und P.schmidtgeni Heller, schließlich Microtus arvalinus Hinton, M.coronensis Kormos und M.ratticepoides Hinton, die insgesamt für eine Einstufung der betr. Bildungen in das Biharium, genauer die Zone 6 der faunenchronologischen Abfolge KrEtzors (1956, S. 217/218) sprechen.

Was nun die genauere zeitliche Einstufung der bei Niederaußem erschlossenen fossilführenden Schichten und damit allenfalls eines mehr oder weniger großen Komplexes des dortigen Quartärprofils anbelangt, so muß zunächst gesagt werden, daß sich aus rein paläontologisch-stratigraphischer Sicht nicht so ohne weiteres bindende Anhaltspunkte ergeben. Das bisherige Fehlen jeglicher Begleitformen macht die überlieferte Faunula von Halsbandlemmingen zu eintönig und zu farblos. Von vornherein läßt sie ferner keinerlei Vergleiche zu mit anderen fossilen Faunengesellschaften aus dem Rheintal, wobei ganz besonders an jene gut bekannten Kleinsäugerfaunen des älteren Quartärs aus den Mosbacher Sanden bei Mainz-Wiesbaden (HELLER 1933), aus den ungefähr gleichalterigen Sanden vom Pilgerhaus bei Weinheim a. d. Bergstraße, sowie den noch wesentlich älteren Schneckenmergeln von Hohensülzen bei Worms (HeLler 1962) zu denken ist.

Das alleinige Vorkommen relativ häufiger Reste eines Halsbandlemmings läßt andererseits nur den Schluß zu, daß während der Bildungszeit der betreffenden Ablagerungen offensichtlich kaltes Klima geherrscht haben muß, wie dies jedenfalls bei massiertem Auftreten dieser so bezeichnenden hocharktischen Elemente auch sonst ganz allgemein und ohne Bedenken angenommen wird.

Ohne Wissen um die beträchtliche Tiefenlage $(33 \mathrm{~m})$ der Fundschicht, in welcher die kleinen Reste in nesterartigen Anreicherungen angetroffen wurden, was auf eine Einbettung ursprünglicher Gewölle zurückzuführen sein dürfte, könnte man geneigt sein, kurzerhand würmzeitliches, und zwar am ehesten hochwürmzeitliches Alter anzunehmen. Dies aber ist bei der geologischen Gesamtsituation der Fundstelle natürlich völlig ausgeschlossen. Dagegen spricht m. E. ferner auch der phyletische Zustand der DicrostonyxForm von Niederaußem, die gegenüber den typischen, wohlbekannten, jungquartären Dicrostonyx-Arten weniger evoluierten, einfacheren Bau der Molaren aufweist, was seine Erklärung nur in höherem geologischen Alter finden kann.

Keine der drei weiter oben bereits angeführten Faunen (Hauptfauna der Mosbacher Sande, Pilgerhaus bei Weinheim und Hohensülzen bei Worms) enthält auch nur die geringste Andeutung eines etwaigen Vorkommens von Lemming-Resten (Lemmus und Dicrostonyx). Nach unseren heutigen Erfahrungen und Kenntnissen dürfte dies bedeuten, daß sie keinesfalls irgendwie geartete Kaltfaunen repräsentieren können, ganz im Gegensatz zu der Halsbandlemming-Fauna des Quartärprofils von Niederaußem. Wie wir mit unseren Ausführungen wohl zur Genüge gezeigt haben, kommt als zeitliche Fixierung 
für letztere mindestens riß-zeitliches Alter in Frage. Unter Berücksichtigung der geologischen Fakten der Rheinterrassen von Niederaußem scheint diese Datierung aber gar nicht auszureichen, so daß wir noch höheres Alter annehmen, also an eine Zuweisung der Fauna in eine davor gelegene Kaltphase denken müssen. Auf alle Fälle beanspruchen die Lemmingfunde von Niederaußem ganz besonderes Interesse, zeigen sie doch, welche Möglichkeiten durch die Entdeckung weiterer Teilfaunen zur noch eingehenderen Gliederung der Quartärbildungen im Rheintale künftiger Forschung vorbehalten und an die Hand gegeben wären.

\section{Schrift tum}

Ahorner, L.: Untersuchungen zur quartären Bruchtektonik der Niederrheinischen Bucht. Eiszeitalter u. Gegenwart 13, 24-105, O'hringen 1962.

Allen, G. M.: Bull. Mus. Comp. Zool. Harvard Coll. 62, Cambridge, Mass. 1919.

BredDin, H.: Die unterirdische Oxydation der Braunkohle im Kölner Revier während der Pleistozän-Zeit. Fortschr. Geol. Rheinl. Westf. 1 u. 2, 683-720, Krefeld 1958.

Brelie, v. D. G.: Probleme der stratigraphischen Gliederung des Pliozäns und Pleistozäns am Mittel- und Niederrhein. Fortschr. Geol. Rheinl. Westf. 4, 371-388, Krefeld 1959.

BrunNaCKer, K.: Altpleistozäne Frostspalten im Gebiet des Hahnbacher Sattels. Geol. Bl. NOBayern 13, 157-163, Erlangen 1963.

Brunnacker, K.; Paulus, Br.; Brockert, M.; Hinsch, W. \& Vidal, H.: Erläuterungen zur Geologischen Karte von Bayern 1:25 000, Blatt Nr. 7736 Ismaning. Bayer. geol. L.A., München 1964.

BrunNer, G.: Eine präglaciale Fauna aus dem Windloch bei Sackdilling (Oberpfalz). N. Jb. Min. Beil. Bd. 71 B, 303-328, Stuttgart 1933. - - Das Osterloch bei Wurmrausch (Oberpfalz). Eine Kleinsäugetierfauna aus dem Mittel-Diluvium mit Spalax sp. Abh. Naturhist. Ges. Nürnberg 26, 2, 24 S., Nürnberg 1936. - - Eine Glazialfauna des mittleren Diluviums aus dem Enzendorfer Loch bei Vorra a. P. Z. deutsch. geol. Ges. 89, 44-51, Berlin 1937. - - Die Hirtenweberhöhle bei Neukirchen (Sulzbach, Opf.). Ein Beitrag zur Tierwelt des mittleren Diluviums. Z. deutsch. geol. Ges. 91, 432-449, Berlin 1939. - - Das Helmloch bei Etzelwang (Obpf.) Fund von Dolomys lenki Heller nebst einigen Beobachtungen zur Morphologie der fränk. Alb. Z. deutsch. geol. Ges. 92, 499-528, Berlin 1940. - - Das Gaisloch bei Münzinghof (Mfr.) mit Faunen aus dem Altdiluvium und aus jüngeren Epochen. N. Jb. Min. Abh. 91 B, 1-34, Stuttgart 1949. - - Die kleine Teufelshöhle bei Pottenstein (Oberfranken). Abh. bayer. Akad. Wiss., math.-naturw. Kl. N. F. 60, 46 S., München 1951. - - Die Markgrabenhöhle bei Pottenstein (Oberfranken). Eine Fauna des Altdiluviums mit Talpa episcopalis Kormos u. a. N Jb. Geol. Paläont. Mh. 1952, 457-471, Stuttgart 1952. - - Das Schmiedberg-Abri bei Hirschbach (Oberpfalz). Paläont. Z. 33, 152-165, Stuttgart 1959.

Dенм, R.: AltpleistocäneSäuger von Schernfeld bei Eichstätt in Bayern. Mitt. Bayer. Staatssamml. Paläont. hist. Geol. 2, 17-61, München 1962.

Dubois, A. et Stehlin, H. G.: La grotte de Cotencher, station moustérienne. Mém. Soc. paléont. suisse 52-53, 292 S., Bâle (Basel), 1933.

Fejfar, O.: The New Species of Field-Mice (Microtinae) in the Bohemian Pleistocene and their Importance for the detailed Stratigraphy. Casopis mineral. geol. 2, 93-101, Praha 1956. - - Die fossilen Vertreter des Genus Sicista Gray, 1827 auf dem Gebiet der ČSR. Casopis mineral. geol. 4, 25-35, Praha 1959.

Heller, Fl.: Eine Forest-Bed-Fauna aus der Sackdillinger Höhle (Oberpfalz). N. Jb. Min. Beil. Bd. 63 B, 247-298, Stuttgart 1930. - - Fossile Kleinfaunenreste aus der Raumgrotte im Hersbrucker Jura. Cbl. Min. 1932 B, 349-362, Stuttgart 1932. - - Die Wühlmäuse der Mosbacher Sande. Notizbl. Ver. Erdkde. u. Hess. Geol. L.-A., (V) 14, 108-116, Darmstadt 1933. - - Eine Forest Bed-Fauna aus der Schwäbischen Alb. Sber. Heidelb. Akad. Wiss., math.-naturw. Kl. 1936, 2. Abh., 29 S., Heidelberg 1936. - - Eine neue altquartäre Wirbeltierfauna von Erpfingen (Schwäbische Alb). N. Jb. Geol. Paläont. Abh. 107, 1-102, Stuttgart 1958/59. - - Zwei altquartäre Kleinsäugerfaunen aus dem Oberrheingebiet: Hohensülzen bei Worms/Rheinhessen und Pilgerhaus bei Weinheim an der Bergstraße/Baden. Mitt. Geol. Staatsinst. Hamburg 31, 461-489, Hamburg 1962. - - Eine altquartäre Wirbeltierfauna des unteren Cromerium aus der nördlichen Frankenalb. N. Jb. Geol. Paläont. Abh. 118, 1-20, Stuttgart 1963. (1963a). - - Ein bedeutsames Quartärprofil in einer Höhlenruine bei Hunas/Hartmannshof (Nördliche Frankenalb). Eiszeitalter u. Gegenwart 14, 111-116, Óhringen/Württ. 1963. (1963b). 
Hinton, M. A. C.: The British fossil Shrews. Geol. Mag. (V) 8, 529-539, London 1911. - - Monograph of the Voles and Lemmings (Microtinae) living and extinct. 1, 488 S., London 1926.

JANossy, D.: Die Entwicklung der Kleinsäugerfauna Europas im Pleistozän (Insectivora, Rodentia, Lagomorpha). Z. Säugetierkd. 26, 1-11, Hamburg u. Berlin 1961.

KaISER, Kh.: Klimazeugen des periglazialen Dauerfrostbodens in Mittel- und Westeuropa. Eiszeitalter u. Gegenwart 11, 121-141, O'Ohringen 1960. - - Gliederung und Formenschatz des Pliozäns und Quartärs am Mittel- und Niederrhein, sowie in den angrenzenden Niederlanden unter besonderer Berücksichtigung der Rheinterrassen. Köln und die RheinlandeFestschr. XXXIII. Deutsch. Geographentag 1961 in Köln, 236-278, Wiesbaden 1961.

Karrenberg, H. \& Quitzow, H. W.: Uber Hohlraumbildungen und Einstürze in Lößböden als Folge unterirdischer Materialwegführungen. Geol. Jb. 71, 631-642, Hannover 1956.

Konmos, Th.: Zur Frage der Abstammung und Herkunft der quartären Säugetierfauna Europas. Festschr. Embrik STRAND 3, 287-328, Riga 1937.

KretzoI, M.: Die altpleistozänen Wirbeltier-Faunen des Villányer Gebirges. Geol. Hungarica, Ser. Palaeont. 27, 1-264, Budapest 1956.

MüCKenhausen, E.: Über die Geschichte der Böden. Geol. Jb. 69, 501-516, Hannover 1955.

Pidoplichko, I. G.: Nowye dannye o faune poswonotschnych anthropogenowych otloschenij Ternopolskoj oblasti. Dokl. Akad. Nauk. SSSR. 100, 989-991, Moskwa-Leningrad 1955.

Quirzow, H. W.: Die Terrassengliederung im Niederrheinischen Tiefland. Geol. en Mijnbouw, N. S. 18, 357-373, s'Gravenhage 1956.

SOERGEL, W.: Der Klimacharakter der als nordisch geltenden Säugetiere des Eiszeitalters. Sber. Heidelb. Akad. Wiss., math.-naturw. Kl. 1941, 4 Abh., 36 S., Heidelberg 1943.

Tillmann, H. \& Kirchock, E.: Neuere Untersuchungen im Braunkohlentertiär der Oberpfalz. Geol. Bavarica 21, München 1954.

VINKEN, R.: Sedimentpetrographische Untersuchung der Rheinterrassen im östlichen Teil der Niederrheinischen Bucht. Fortschr. Geol. Rheinl. u. Westf. 4, 127-170, Krefeld 1959.

Manuskr. eingeg. 20. 2. 1966.

Anschrift der Verf.: Prof. Dr. Fl. Heller, Geol. Inst. der Univ., 852 Erlangen, Schloßgarten 5; Prof. Dr. K. Brunnacker, Geol. Inst. der Univ., 5 Köln, Zülpicher Straße 49. 